\title{
Implante de marcapaso definitivo en pacientes con vena cava superior izquierda persistente y ausencia de vena cava superior derecha. Caso clínico
}

\author{
Patricia Frangini S, Ismael Vergara S, Rolando González A, \\ Alejandro Fajuri N, Pablo Casanegra P. \\ Permanent pacemaker implantation \\ in patients with persistent left superior \\ vena cava and absent right superior \\ vena cava. Report of three cases
}

Persistent left superior vena cava and absent right superior vena cava is an uncommon anatomical association. This is a challenging situation for permanent pacemaker implantation. We report three patients with this anomaly and a permanent pacemaker successfully implanted through the left superior vena cava and coronary sinus, without acute or chronic complications (Rev Méd Chile 2006; 134: 767-71).

(Key words: Coronary sinus; Pacemaker, artificial; Vena cava superior)

Recibido el 19 de agosto, 2005. Aceptado en versión corregida el 22 de noviembre, 2005. Laboratorio de Electrofisiología Cardíaca, Departamento de Enfermedades Cardiovasculares, Facultad de Medicina, Pontificia Universidad Católica de Chile.

L a vena cava superior izquierda persistente es una variante anatómica conocida con una incidencia de 1-2\% en la población general. En pacientes portadores de cardiopatías congénitas la prevalencia es más alta, llegando a $11 \%$ en tetralogía de Fallot, 19\% en canal aurículo-ventricular, $17 \%$ en atresia mitral y $34 \%$ en yuxtaposición de la orejuela derecha ${ }^{1,2}$. Su persistencia se produce porque falla la involución de las venas cardinal común izquierda y anterior izquierda ${ }^{3}$. La

Correspondencia a: Dr. Ismael Vergara S. Marcoleta 367, 2o piso. Teléfono: 3546402. Fax: 6327306. E mail: ivergara@med.puc.cl vena cava superior izquierda persistente puede ser única (ausencia de cava superior derecha, asociación mucho menos frecuente) o pueden existir ambas venas cavas, comunicadas o no por una vena innominada. En $92 \%$ de los casos la vena cava superior izquierda persistente drena a la aurícula derecha a través del seno coronario, y en el resto de los pacientes drena a la aurícula izquierda a través de un seno coronario destechado. La asociación entre esta alteración anatómica y enfermedad del sistema éxcito-conductor no ha sido evaluada.

Presentamos tres pacientes portadores de vena cava superior izquierda persistente y ausencia de vena cava superior derecha, sin cardiopatía asocia- 
da, sometidos exitosamente a implante de marcapaso definitivo utilizando este acceso venoso.

\section{CASO 1}

Mujer de 61 años, con antecedente de enfermedad del nódulo sinusal sintomática y fibrilación auricular paroxística, por lo cual se le indicó implante de marcapaso definitivo bicameral. Se intentó implante del marcapaso por acceso subclavio izquierdo, pero al avanzar la guía se observó que ésta seguía un trayecto anormal, avanzando en sentido descendente y paravertebral izquierdo. La inyección de medio de contraste confirmó la existencia de vena cava superior izquierda. Se decidió intentar el implante por la vena subclavia derecha, el cual fracasó debido a ausencia de vena cava superior derecha. Se interrumpió el procedimiento y se discutió con la paciente la posibilidad de implantar el marcapaso con electrodos epicárdicos o reintentar el implante a través de la vena cava superior izquierda persistente. Se optó por la segunda alternativa, y en el laboratorio de electrofisiología se realizó doble punción de la vena axilar izquierda y, a través de introductores 8 French, se avanzó dos electrodos rectos de fijación activa (Saint Jude ${ }^{\circledR}$ modelo 1488T), vía vena cava superior izquierda y seno coronario hasta la vena cava inferior. El electrodo ventricular se avanzó hasta la aurícula derecha y se realizó un asa hacia el anillo tricuspídeo, avanzando el electrodo hasta la región basal del ventrículo derecho donde fue posicionado, obteniendo una onda $\mathrm{R}$ sensada de $6,0 \mathrm{mV}$, un umbral de estimulación ventricular de 1,0 Volt, con impedancia de estimulación de 630 Ohms. El electrodo auricular se posicionó en la cara lateral de la aurícula derecha, donde se obtuvo una onda $P$ sensada de $5,4 \mathrm{mV}$, un umbral de estimulación auricular de 0,6 Volt, con impedancia de estimulación de 500 Ohms (Figura 1). No hubo complicaciones asociadas al procedimiento y la paciente fue dada de alta a las $24 \mathrm{~h}$. Los controles posteriores han mostrado que los parámetros de funcionamiento se han mantenido estables a 2 años de seguimiento.

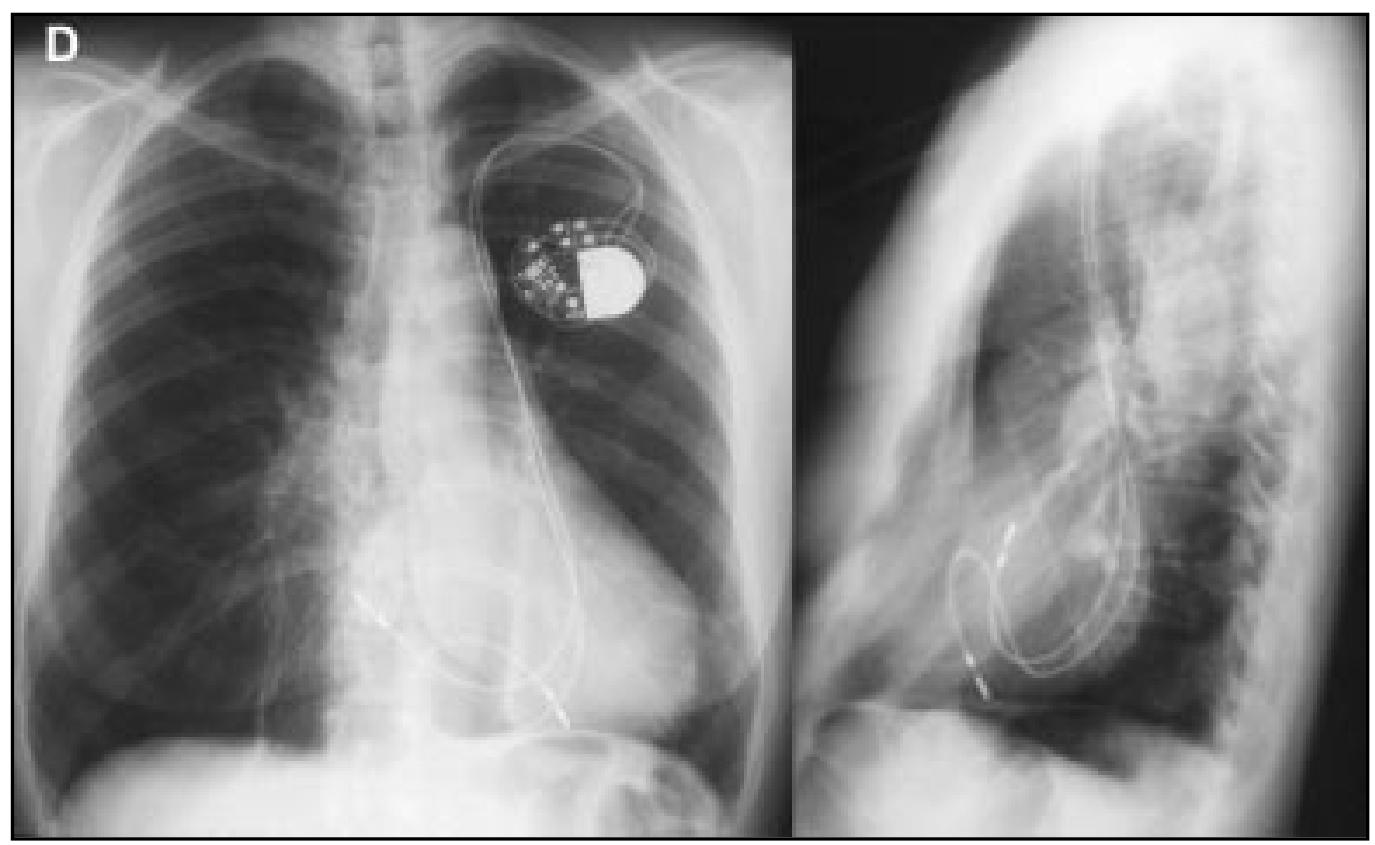

Figura 1. Radiografía de tórax anteropostenior y lateral. Se observa el generador de pulso en la región subclavicular izquierda y dos electrodos de fijación activa con trayecto descendente paravertebral izquierdo a través de la vena cava superior izquierda persistente hasta la aurícula derecha, vía seno coronario. El electrodo auricular está implantado a nivel de cara lateral de la aunícula y el electrodo ventricular forma un asa hacia la base del ventrículo derecho. 


\section{CASO 2}

Hombre de 49 años, con taquicardia auricular sintomática y refractaria a tratamiento antiarrítmico. Se realizó estudio electrofisiológico que demostró flutter auricular izquierdo y asociación de vena cava superior izquierda persistente, drenando a la aurícula derecha a través del seno coronario y ausencia de vena cava superior derecha. Se discutió con el paciente la posibilidad de intentar fulguración de su arritmia con una tasa no despreciable de fracaso y recidiva, versus una forma de tratamiento sintomático definitivo mediante la fulguración del nodo aurículo-ventricular e implante de marcapaso ventricular VVI-R. El implante del marcapaso se realizó a través de punción de la vena axilar izquierda, avanzando el electrodo Saint Jude ${ }^{\circledR}$ modelo $1488 \mathrm{~T}$ a través de la vena cava superior izquierda y seno coronario, hasta la aurícula derecha. Se avanzó el electrodo a nivel del tracto de salida del ventrículo derecho y, finalmente se posicionó en el ápex del ventrículo (Figura 2), donde se obtuvo una onda R sensada de $14,0 \mathrm{mV}$, un umbral de estimulación ventricular de 0,5 Volt, con impedancia de estimulación de 650 Ohms. Posterior al implante se realizó fulguración del nodo aurículo-ventricular. No hubo complicaciones y fue dado de alta al día siguiente. Sus controles posteriores han mostrado parámetros normales a un año y 10 meses de seguimiento, con alivio total de sus síntomas, sin tratamiento antiarrítmico.

\section{CASO 3}

Hombre de 78 años, con antecedentes de hipertensión arterial, diabetes mellitus y enfermedad de Parkinson. Se hospitalizó por bloqueo aurículoventricular 2x1 y bloqueo aurículo-ventricular completo paroxístico, con síncope, por lo que se le indicó implante de marcapaso definitivo, optándose por razones económicas por un marcapaso ventricular VVI. Se realizó el bolsillo subcutáneo prepectoral izquierdo y se puncionó la vena axilar izquierda como habitualmente se realiza en nuestro laboratorio, bajo visión radioscópica durante venograma, el que demostró una vena cava superior izquierda persistente drenando hacia el seno coro-

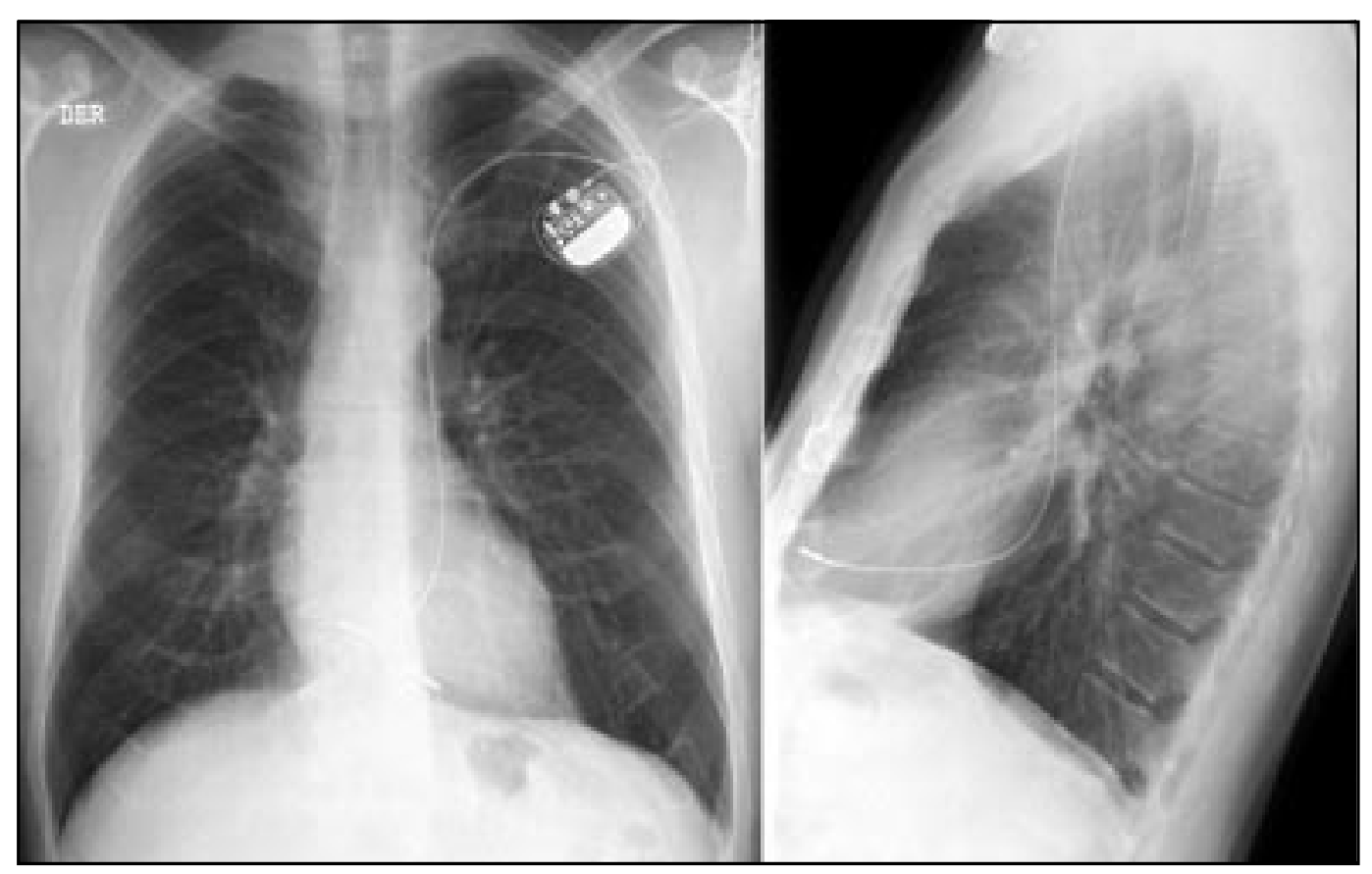

Figura 2. Radiografía de tórax anteroposterior y lateral. El generador de pulso unicameral está implantado en la región subclavicular izquierda. Un electrodo de fijación activa se implantó a nivel septoapical del ventrículo derecho, vía vena cava superior izquierda persistente y seno coronario. 
nario. A través de introductor 7 French, se avanzó el electrodo ventricular de fijación activa Saint Jude ${ }^{\circledR}$ modelo $1688 \mathrm{~T}$ hasta la aurícula derecha. Luego se avanzó el electrodo hasta el tracto de salida del ventrículo derecho y se posicionó a nivel septoinfundibular del ventrículo derecho (Figura $3)$, donde se obtuvo una onda $R$ sensada de 8,0 $\mathrm{mV}$, un umbral de estimulación ventricular de 0,6 Volt, con impedancia de estimulación de 650 Ohms. Se demostró ausencia de vena cava superior derecha mediante inyección de medio de contraste en la vena braquial derecha. El paciente no presentó complicaciones y fue dado de alta a las 24 h. Sus controles posteriores han mostrado funcionamiento normal de su marcapaso con parámetros adecuados y estables, después de un año.

\section{DiscUSIÓN}

La vía de abordaje más utilizada para implante de electrodos endocavitarios en marcapasos definitivos es el sistema venoso subclavio izquierdo y sus venas tributarias. En algunas ocasiones se planea con anticipación el implante por el sistema venoso derecho, por ejemplo, en pacientes zurdos, en pacientes portadores de marcapaso previo con antecedente de infección del bolsillo, pacientes con antecedente de trombosis venosa de la extremidad superior izquierda, o pacientes con antecedente de alguna condición que haga sospechar obstrucción del sistema venoso izquierdo (ej: radioterapia y/o mastectomía radical por cáncer de mama izquierdo). Sin embargo, es excepcional poder anticipar la presencia de una vena cava superior izquierda persistente en pacientes con corazón estructuralmente normal antes del implante del marcapaso, y sólo se detecta esta anomalía al observar un curso inhabitual de la guía descendiendo por el lado izquierdo del tórax después de puncionar la vena subclavia o axilar izquierda. Este hallazgo se puede confirmar realizando un venograma que permite observar que el medio de contraste drena a la aurícula derecha a través del seno coronario, sin producirse cortocircuito intracardíaco, como ocurrió en el caso de los tres pacientes presentados. En ocasiones el drenaje de esta vena es a la aurícula izquierda a través de un seno coronario destechado $\mathrm{y}$, en este caso se produce cortocircuito intracardíaco y la saturación del paciente es anormal.

Cuando uno se encuentra con este hallazgo en un paciente al cual se le está implantando un

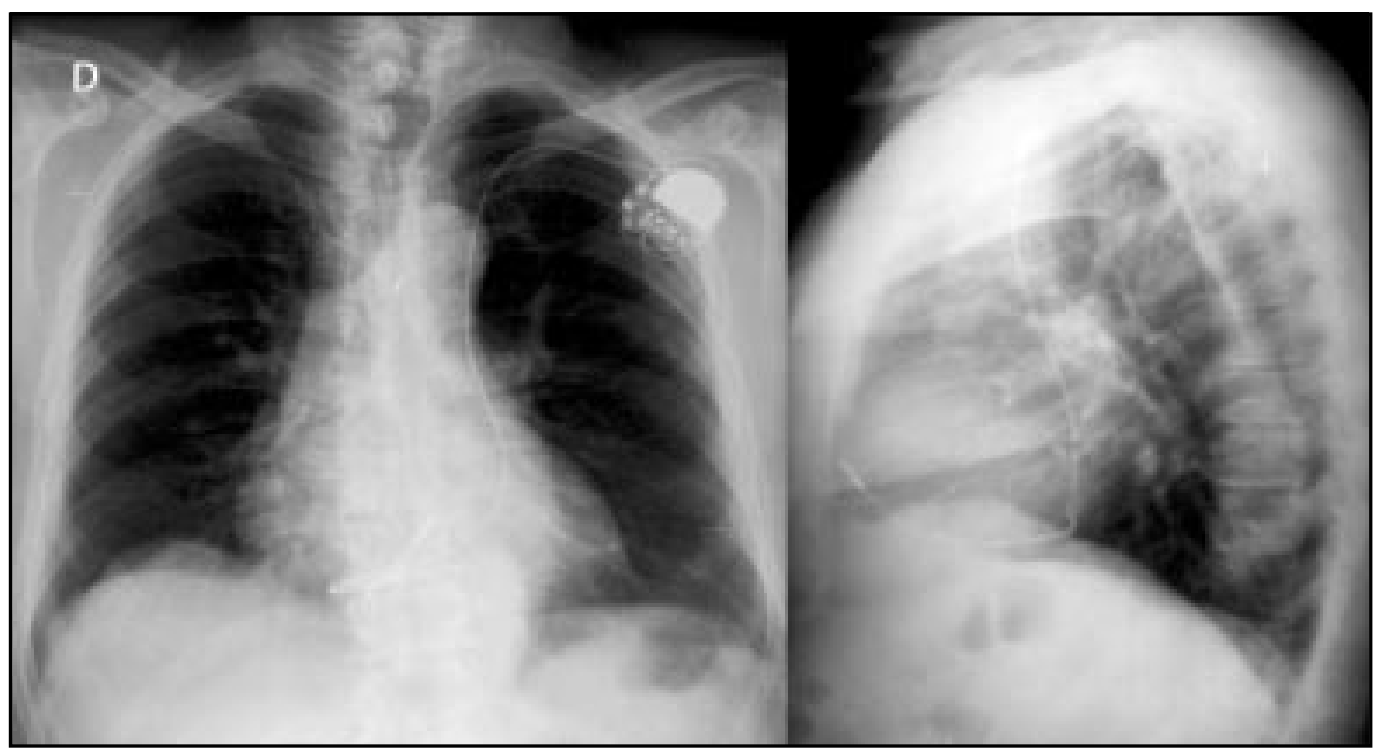

Figura 3. Radiografía de tórax anteroposterior y lateral. Se observa el generador de pulso unicameral en la región prepectoral izquierda y el electrodo de fijación activa posicionado en la región septoinfundibular del ventrículo derecho, ingresando al corazón a través de la vena cava superior izquierda persistente y seno coronario. 
marcapaso, por lo general, ya se ha confeccionado el bolsillo subcutáneo donde se colocará el generador del marcapaso, y las opciones a las que se ve enfrentado el operador son abortar el implante por ese lado e intentarlo por el sistema venoso derecho, proseguir con el implante a través de la vena cava superior izquierda y estimular las cavidades derechas o intentar estimulación ventricular izquierda, avanzando el electrodo hacia alguna vena coronariana. En los casos reportados en esta serie no era posible el abordaje por el lado derecho dada la ausencia de vena cava superior derecha, y tal como ocurrió en uno de los pacientes, frente al fracaso del implante desde ambos lados, en algunos casos se ha sugerido el uso de electrodos de estimulación epicárdicos ${ }^{4}$. Existen varios casos reportados de pacientes portadores de esta anomalía en quienes se ha realizado el implante de marcapaso definitivo utilizando este acceso venoso sin incovenientes ${ }^{5}$. Las publicaciones incluyen no sólo sistemas de estimulación uni o bicamerales ${ }^{5-8}$, sino también sistemas biventriculares ${ }^{9}$. Además, a través de la vena cava superior izquierda es posible manipular

\section{REFERENCIAS}

1. Fraser RS, Dvorkin J, Rosall RE, Eidem R Left superior vena cava: a review of associated congenital heart lesions, catheterization data and roentgenologic findings. Am J Med 1961; 31: 711-16.

2. Geva T, Van Praagh S. Abnormal systemic venous connections. En: Moss and Adams', Heart Diseases in Infants, Children, and Adolescents, Sixth edition. Philadelphia, Editorial Lippincott Williams \& Wilkins, 2001; 773-796.

3. NSAH EN, Moore GW, Hutchins GM. Pathogenesis of persistent left superior vena cava with a coronary sinus connection. Pediatr Pathol 1991; 11: 261-69.

4. Lennox CC, Zuberbuhler JR, Park SC, Neches WH, MatHews RA, FRICKER FJ ET AL. Absent right superior vena cava with persistent left superior vena cava: Implications and management. Am J Cardiol 1980; 45: 117-22.

5. Trigano JA. Permanent pacing through a persistent left superior vena cava in 39 collected cases. Clin Prog Electrophysiol Pacing 1986; 3: 45-52.

6. Robbens EJ, Ruiter JH. Atrial pacing via unilateral adecuadamente los electrodos y posicionarlos en el lugar que el operador estime conveniente, lo cual es especialmente importante en el caso del electrodo ventricular derecho, debido a la tendencia actual de posicionar el electrodo a nivel septal o septoinfundibular debido a lo deletéreo que puede resultar la estimulación ventricular derecha apical ${ }^{7}$. No debe olvidarse que en la manipulación de catéteres en el seno coronario está descrito el riesgo de disección, perforación y de trombosis ${ }^{10}$.

Los casos mostrados en nuestra serie confirman que en pacientes portadores de esta anomalía es posible realizar el implante de marcapaso definitivo utilizando la vena cava superior izquierda persistente, tanto en sistemas de estimulación unicameral como bicameral, pudiendo posicionar el electrodo ventricular derecho tanto en el ápex como en la región septoinfundibular, sin mayor número de complicaciones asociadas, con parámetros adecuados en el implante y estables en el tiempo. Para poder mantener el electrodo en posiciones más inestables, nosotros, al igual que otros autores, recomendamos el uso de electrodos de fijación activa ${ }^{11}$.

persistent left superior vena cava. Pacing Clin Electrophysiol 1986; 9: 594-6.

7. Kheng-Siang NG, Foo D, Lim J. His-bundle pacing in a patient with persistent left superior vena cava. Pacing Clin Electrophysiol 2005; 28: 588-90.

8. Birnie D, TANG A. Permanent pacing from a left ventricular vein in a patient with persistent left and absent right superior vena cava. Pacing Clin Electrophysiol 2000; 23: 2135-7.

9. Paulussen GMC, Gelder BMV. Implantation of a biventricular pacing system in a patient with a persistent left superior vena cava. Pacing Clin Electrophysiol 2004; 27: 1014-16.

10. Chatthiraphan S, Goldberg E, Wolff W, Jootar P, Grossman W. Massive thrombosis of the coronary sinus as an unusual complication of transvenous pacemaker insertion in a patient with persistent left, and no right superior vena cava. J Am Geriatr Soc 1974; 22: 79-85.

11. ANTONEш D, Rosenfeld T. Implantation of dual chamber pacemaker in a patient with persistent left superior vena cava. Pacing Clin Electrophysiol 1997; 20: 1737-8. 